\title{
Actividad antifúngica in vitro de los extractivos naturales de especies latifoliadas de la Guayana Venezolana
}

\author{
Jesús Velásquez ${ }^{1}$, María Elena Toro ${ }^{1}$, \\ Luis Rojas ${ }^{2}$, Osvaldo Encinas ${ }^{3}$
}

\begin{abstract}
RESUMEN
Se determinó en condiciones de laboratorio el efecto microbiológico de los metabolitos secundarios, desarrollados como mecanismo de defensa pasiva, en la madera de las especies Puy (Tabebuia serratifolia (Vahl.) Nicholson), Zapatero (Peltogyne porphyrocardia Griseb.), Algarrobo (Hymenaea courbaril L.) y Cartan (Centrolubium paraense Tul. var. orinocense Benth), frente a dos de los más comunes microorganismos que causan deterioro de la madera $G$. traveum y $T$. versicolor. E ensayo microbiológico se realizó empleando el método de dilución en gel con inoculación superficial en placa. Se evaluaron tres tipos de solventes de diferente polaridad para la recuperación de los extractivos (acetona, etanol y agua). Se empleó agar extracto de malta como medio de cultivo a tres niveles de concentración de extractivo $(0,05 \%, 0,1 \%$ y $0,2 \% \mathrm{v} / \mathrm{v})$. La inhibición del crecimiento, fue utilizada como una medida para evaluar la efectividad del producto químico natural. Los resultados obtenidos de la evaluación preliminar demostraron que todos los extractos evaluados presentan actividad biológica asociada con los microorganismos de prueba. El perfil de la actividad antifúngica del extracto soluble en agua, sostiene que es el más tolerable por los microorganismos que deterioran la madera, posiblemente atribuido a la presencia de materiales orgánicos señalados como azúcares del duramen. Los extractos acetónicos y etanólicos demostraron tener la capacidad de extraer la mayor cantidad de compuestos biológicamente activos del duramen de las especies evaluadas, al producir elevados niveles de control en el crecimiento de los microorganismos empleados en el estudio.
\end{abstract}

PALABRAS CLAVE:

Actividad antifúngica, duramen, extractivos, metabolitos secundarios, productos naturales.

\section{ABSTRACT}

The microbiological effect of the secondary metabolites developed as mechanism of passive defense, in the wood of the species Puy (Tabebuia serratifolia (Vahl.) Nicholson), Zapatero (Peltogyne porphyrocardia Griseb.), Algarrobo (Hymenaea courbaril L.) and Cartan (Centrolubium paraense Tul. var. orinocense Benth) was evaluated under laboratory conditions, against two common microorganisms that cause deterioration on the wood G. traveum and T. versicolor. Antifungal assay was carried out using the agar dilution method with superficial inoculation. Solvents, like acetone, ethanol and water, of different polarity were evaluated for the recovery of the extractives. Malt extract agar was used in the antifungal assay at concentration of $0,05 \%, 0,1 \%$ and $0,2 \%(v / v)$. The growth inhibition was used as a measure to evaluate the effectiveness of the natural chemical product. Results of the preliminary evaluation demonstrated that all the evaluated extracts present biological activity associated with the microorganisms test. The antifungal activity of the soluble extract in water, sustains that it is the most tolerant for the microorganisms that deteriorate wood. This may be attributed to the presence of organic materials called heartwood's sugar. The acetonic and ethanolic extracts showed to have the capacity to extract the higher quantity of active biologically compound of the heartwood of the species, producing higher growth inhibition of the microorganisms used in the study. 
KEY WORDS:

Antifungal activity, heartwood, extractives, secondary metabolites, natural products.

\section{INTRODUCCIÓN}

La pudrición de la madera es una de las mayores causas de deterioro microbiológico que ocasiona fallas estructurales de manera rápida, sobre elementos de madera en servicio (Mora y Encinas, 2001). Los más importantes y potentes organismos de pudrición de la madera, son los hongos de pudrición blanca, marrón, blanda y bacterias. La pudrición marrón es la más común y el tipo más destructivo de pudrición de madera en servicio (Eaton y Hale, 1993). Muchas de las especies latifoliadas que presentan una transición abrupta albura-duramen, poseen una particular resistencia, o mejor aún, una biorresistencia al deterioro por la acción de hongos, bacterias e insectos. Esta resistencia se encuentra generalmente atribuida a la presencia, en las paredes celulares de la madera, de sustancias químicas activas que juegan un rol importante en la durabilidad natural. Estas sustancias proporcionan una barrera química con propiedades fungicidas o fungistáticas, que eliminan 0 inhiben el crecimiento y desarrollo de los agentes xilófagos (Deon, 1983; Suttie y Orsler, 1996; Harju et al., 2003). El uso de estos extractivos naturales para el control de microorganismos que deterioran la madera ha sido estudiado como una atractiva alternativa para la protección biológica de la misma, cuya principal ventaja es la de proveer productos naturales efectivos, de baja toxicidad a los seres humanos y ambientalmente aceptables (Velásquez, 2000). En el futuro, es posible llegar a remplazar las propiedades de las formulaciones de preservantes sintéticos para madera, con formulaciones basadas en ingredientes bioactivos naturales, presentes en los extractos tóxicos de la madera o de otros componentes químicos resistentes, aislados de productos naturales (Wong y Singh, 1997). Es relativamente amplia la literatura relacionada con la evaluación de la durabilidad natural e inducida de la madera (Eaton y Hale, 1993; Winandy y Morel, 1993; Troya et al., 1999; Curling et al., 2000; Kennedy et al., 2000; Papinutti et al., 2003; Gierlinger et al., 2004; Velásquez et al., 2004; Uzcátegui et al., 2004; Mora et al., 2004). Sin embargo, puede considerarse limitada la literatura que relaciona el efecto de los extractivos naturales de especies venezolanas, sobre el control de los microorganismos que causan el deterioro de los componentes estructurales de la pared celular de la madera.

\section{OBJETIVO}

El objetivo del presente estudio fue evaluar la acción fungicida de los extractos o productos naturales del duramen de cuatro especies forestales latifoliadas de durabilidad natural elevada provenientes de los bosques de la Región Guayana, en Venezuela (IFLA, 1987; Mora y Encinas, 2001; Velásquez et al., 2004), frente a dos de los microorganismos más comunes que causan el deterioro en la madera: Gloeophylum trabeum (pudrición marrón) y Trametes versicolor (pudrición blanca).

\section{METODOLOGÍA}

\section{Material vegetal}

Se emplearon muestras del duramen de cuatro especies latifoliadas, comúnmente explotadas en la región Guayana, Edo. Bolívar, Venezuela. Para realizar la recuperación de los extractos, se tomaron muestras de duramen en la base de los árboles de las especies Puy 
(Tabebuia serratifolia (Vahl.) Nicholson), Zapatero (Peltogyne porphyrocardia Griseb.), Algarrobo (Hymenaea courbaril L.) y Cartan (Centrolubium paraense Tul. var. orinocense Benth).

\section{Microorganismo de ensayo}

Se emplearon dos hongos filamentosos, uno representante del efecto de deterioro conocido como pudrición marrón Gloeophylum trabeum (Fr.) Murr. (Mad-617-R) y un representante de los hongos de pudrición blanca Trametes versicolor (L:Fr) Pilát (FP-133255-R). Ambos fueron seleccionados por su conocida capacidad como agentes destructores de madera, de importancia económica y de empleo común en evaluaciones de preservadores para madera. Los microorganismos fueron suministrados por la sección de preservación de maderas del Laboratorio Nacional de Productos Forestales, de la Universidad de Los Andes, Mérida, Venezuela. Estos se encontraban desarrollados en cápsulas de Petri sobre agar extracto de malta al 2,5\% y mantenidos en refrigeración $\left(4^{\circ} \mathrm{C}\right)$. Para la prueba de la actividad microbiológica, los hongos se activaron sembrándolos en cápsulas de Petri al $2,5 \%$ de agar extracto de malta e incubados por seis días a $26 \pm 2{ }^{\circ} \mathrm{C}$.

\section{Preparación de los extractos}

Las muestras de duramen fueron molidas y tamizadas hasta obtener un tamaño de partículas adecuado para el proceso de extracción (40-60 mesh), basado en la norma TAPPI T 264 om-97 (1998). Para obtener los extractos, se empaquetaron $30 \mathrm{~g}$ de material lignocelulósico molido por cada especie, y se colocaron en reflujo en extractores Soxhlet, empleando como solventes acetona y etanol (95\%). La extracción con cada solvente se realizó con muestras nuevas y por separado para cada tipo de madera. El extracto en agua se recuperó de acuerdo con lo establecido por la norma TAPPI T 207 om-93 (1998). La mezcla solventeextractivo recuperada y filtrada en cada proceso de extracción, fue concentrado hasta un residuo viscoso, bajo presión reducida a una temperatura no mayor de $50{ }^{\circ} \mathrm{C}$, luego se colocó en estufa por una hora a $50 \pm 2{ }^{\circ} \mathrm{C}$. El residuo sólido obtenido fue disuelto completamente en $30 \mathrm{ml}$ de etanol $(95 \%)$ y mantenido en refrigeración $\left(4{ }^{\circ} \mathrm{C}\right)$, en botellas ámbar hasta el momento de su uso.

\section{Ensayo microbiológico}

La evaluación de la actividad antifúngica de los diferentes extractos obtenidos se realizó por el método de dilución en gel con inoculación superficial en placa (Jansser et al.,1987; García et al.,1995; Ajaiyeoba et al.,1998). En cada cápsula de Petri de diez centímetros de diámetro, se vertieron $20 \mathrm{ml}$ de agar extracto de malta con el extracto incorporado, obteniéndose una concentración final del medio de 0,05\%, 0,1\% y $0,2 \%$ $(\mathrm{v} / \mathrm{v})$. Simultáneamente, se adicionó etanol (95\%) al medio de cultivo el cual funcionó como blanco o control. El nuevo medio de cultivo, con el extracto incorporado, se dejó solidificar a temperatura ambiente durante una hora y posteriormente fue inoculado, colocando sobre éste, y en el centro de cada cápsula, un inóculo circular del microorganismo de prueba $(10 \mathrm{~mm})$, e incubadas durante seis días a $26 \pm 2{ }^{\circ} \mathrm{C}$, tiempo en el cual el testigo cubre completamente la cápsula de Petri. Las determinaciones se realizaron por triplicado para cada concentración, extracto y hongo de prueba. El crecimiento diametral de cada microorganismo $(\mathrm{mm})$ fue medido al final del periodo de incubación (6 días), y el porcentaje de inhibición fue expresado en función del crecimiento total del control, de acuerdo 
con la metodología señalada por Gopalakrishnam et al. (1997).

\section{RESULTADOS Y DISCUSIÓN}

La tabla 1 muestra la cuantificación porcentual de los extractivos recuperados, a partir de las muestras del duramen de las cuatro especies forestales evaluadas (Algarrobo, Cartan, Puy, y Zapatero), empleando como solventes acetona, etanol y agua.

En estos resultados se observa, que las especies Algarrobo, Cartan y Zapatero, presentaron la mayor proporción de extracto recuperados con los diferentes solventes empleados. El extracto recuperado con etanol mostró la mayor producción de extractivo entre las especies evaluadas $(15,2 \%(A), \quad 16,5 \%(B) \quad y$ $10,9 \%(C))$, con excepción de la especie Zapatero, cuyo mayor porcentaje estuvo representado por el extracto soluble en agua $(14,68 \%$ del peso seco).

Los resultados obtenidos de la evaluación preliminar sobre la actividad microbiológica de los diferentes extractos, empleando el método de dilución en gel con inoculación superficial en placa, después de seis días de incubación, se observan en la tabla 2 . Estos resultados evidencian que todos los extractos de las especies evaluadas presentan actividad biológica asociada con $T$. versicolor y G. trabeum, indicando además, que los principios activos tóxicos presentes en el duramen varían con la especie y con el tipo o sistema de solvente empleado en la recuperación de los extractivos. Sin embargo, solo los extractos etanólicos y acetónicos recuperados de la especie Puy fueron más tóxicos, inclusive a niveles de concentración más bajos $(0,05 \%)$, con un porcentaje de inhibición de crecimiento del 85 y $94 \%$, respectivamente para $G$. trabeum, y 78-83\%, para T. versicolor. Estos resultados confirman lo reportado en investigaciones previas, en las cuales se plantean que los extractivos del duramen muestran propiedades fungicidas que contribuyen con las propiedades de biorresistencia y durabilidad natural de cada especie (Deon, 1983; Schultz y Darrel, 1997; Bhargava et al., 1998).

No se observó una relación directa entre el contenido de extractivo soluble en acetona, etanol y agua del duramen de las diferentes especies evaluadas (en un rango $3,5 \%-16,57 \%$ ) y la inhibición del crecimiento de los microorganismos de prueba. Estos resultados difieren de otras investigaciones, como la desarrollada por Viitanen et al. (1997), quienes encontraron una relación directa entre la resistencia de la madera y el contenido de extractivos en diversas especies de

Tabla 1. Cuantificación de extractivos (\%) con base en el peso seco de la madera

\begin{tabular}{lccc}
\hline ESPECIES & \multicolumn{3}{c}{ SOLVENTES } \\
\cline { 2 - 4 } & ACETONA (\%) & ETANOL (\%) & AGUA (\%) \\
\hline Algarrobo (A) & 11,0 & 15,24 & 13,48 \\
Cartan (B) & 11,0 & 16,57 & 8,68 \\
Puy (C) & 6,85 & 10,96 & 3,50 \\
Zapatero (D) & 7,10 & 11,43 & 14,68 \\
\hline
\end{tabular}


Larix en un rango de $3,2 \%-20,5 \%$, demostrando que a menores niveles de extractivos menor es la resistencia de esta madera, y viceversa. Esta falta de relación entre la resistencia al deterioro y el contenido de extractivo puede ser atribuida a la composición química intrínseca de cada especie y de cada extracto en particular (elevado nivel de compuestos que muestran actividad fungicida sobre los hongos de deterioro).

En los resultados resumidos en la tabla 2 se evidencian diferentes niveles de respuestas de los microorganismos de prueba frente al nuevo medio de cultivo (con extractos), para ser empleado como fuente de carbono. Este comportamiento, puede ser atribuido a la diferencias enzimáticas y metabólicas existentes entre ambos hongos de pudrición (marrón y blanca). Estos tienen la propiedad de producir una variedad de enzimas específicas para promover la degradación del sustrato, y luego una incorporación de estos productos de degradación a los ciclos metabólicos de los microorganismos, dentro de las que se señalan celulasas, ligninasas, lacasa, manganeso peroxidasa, entre otras (Goodell et al., 1997; Papinutti et al., 2003).

Tabla 2. Valor medio y desviación estándar de la inhibición del crecimiento (\%) de los extractos recuperados con acetona, etanol y agua después de seis días de incubación

\begin{tabular}{|c|c|c|c|c|c|c|c|c|c|}
\hline & \multicolumn{5}{|c|}{ Gloeophylum trabeum } & \multicolumn{4}{|c|}{ Trametes versicolor } \\
\hline Solvente & \% \% & Algar. & "Cartan & Puy & Zapat. & Algar. & "Cartan & Puy & Zapat. \\
\hline \multirow{3}{*}{ Acetona } & 0,05 & $\begin{array}{c}16 \\
(4,21)\end{array}$ & $\begin{array}{c}\mathbf{6 2} \\
(2,25)\end{array}$ & $\begin{array}{c}85 \\
(2,07)\end{array}$ & $\begin{array}{c}10 \\
(3,55)\end{array}$ & $\begin{array}{c}35 \\
(5,62)\end{array}$ & $\begin{array}{c}\mathbf{3 9} \\
(6,78)\end{array}$ & $\begin{array}{c}\mathbf{7 8} \\
(3,56)\end{array}$ & $\begin{array}{c}\mathbf{1 8} \\
(1,19)\end{array}$ \\
\hline & 0,10 & $\begin{array}{c}\mathbf{1 8} \\
(2,11)\end{array}$ & $\begin{array}{c}73 \\
(1,92)\end{array}$ & $\begin{array}{c}\mathbf{9 2} \\
(2,41)\end{array}$ & $\begin{array}{c}17 \\
(1,91)\end{array}$ & $\begin{array}{c}\mathbf{6 2} \\
(2,54)\end{array}$ & $\begin{array}{c}73 \\
(2,31)\end{array}$ & $\begin{array}{c}90 \\
(0,75)\end{array}$ & $\begin{array}{c}\mathbf{4 0} \\
(2,75)\end{array}$ \\
\hline & 0,20 & $\begin{array}{c}\mathbf{3 7} \\
(1,08)\end{array}$ & $\begin{array}{c}\mathbf{9 3} \\
(0,79)\end{array}$ & $\begin{array}{c}\mathbf{9 6} \\
(1,38)\end{array}$ & $\begin{array}{c}\mathbf{2 3} \\
(2,82)\end{array}$ & $\begin{array}{c}\mathbf{6 7} \\
(2,89)\end{array}$ & $\begin{array}{c}81 \\
(3,46)\end{array}$ & $\begin{array}{c}\mathbf{9 0} \\
(2,51)\end{array}$ & $\begin{array}{c}\mathbf{4 0} \\
(1,08)\end{array}$ \\
\hline \multirow{3}{*}{ Etanol } & 0,05 & $\begin{array}{c}\mathbf{2 9} \\
(3,32)\end{array}$ & $\begin{array}{c}69 \\
(1,14)\end{array}$ & $\begin{array}{c}\mathbf{9 4} \\
(0,60)\end{array}$ & $\begin{array}{c}\mathbf{3 6} \\
(4,11)\end{array}$ & $\begin{array}{c}65 \\
(2,01)\end{array}$ & $\begin{array}{c}\mathbf{6 7} \\
(5,16)\end{array}$ & $\begin{array}{c}\mathbf{8 3} \\
(0,23)\end{array}$ & $\begin{array}{c}22 \\
(6,28)\end{array}$ \\
\hline & 0,10 & $\begin{array}{c}44 \\
(2,56)\end{array}$ & $\begin{array}{c}80 \\
(1,22)\end{array}$ & $\begin{array}{c}96 \\
(1,70)\end{array}$ & $\begin{array}{c}\mathbf{6 0} \\
(3,45)\end{array}$ & $\begin{array}{c}75 \\
(6,91)\end{array}$ & $\begin{array}{c}76 \\
(3,68)\end{array}$ & $\begin{array}{c}95 \\
(1,65)\end{array}$ & $\begin{array}{c}39 \\
(8,12)\end{array}$ \\
\hline & 0,20 & $\begin{array}{c}70 \\
(0,87)\end{array}$ & $\begin{array}{c}100 \\
(0,69)\end{array}$ & $\begin{array}{c}100 \\
(0,30)\end{array}$ & $\begin{array}{c}\mathbf{6 5} \\
(1,06)\end{array}$ & $\begin{array}{c}79 \\
(0,59)\end{array}$ & $\begin{array}{c}\mathbf{9 2} \\
(1,57)\end{array}$ & $\begin{array}{c}\mathbf{9 7} \\
(1,21)\end{array}$ & $\begin{array}{c}55 \\
(4,11)\end{array}$ \\
\hline \multirow{3}{*}{ Agua } & 0,05 & $\begin{array}{c}\mathbf{0} \\
(0,35)\end{array}$ & $\begin{array}{c}\mathbf{8} \\
(2,21)\end{array}$ & $\begin{array}{c}\mathbf{5} \\
(3,50)\end{array}$ & $\begin{array}{c}\mathbf{0} \\
(0,12)\end{array}$ & $\begin{array}{c}\mathbf{0} \\
(0.11)\end{array}$ & $\begin{array}{c}\mathbf{0} \\
(1,35)\end{array}$ & $\begin{array}{c}\mathbf{0} \\
(0,26)\end{array}$ & $\begin{array}{c}\mathbf{0} \\
(0,23)\end{array}$ \\
\hline & 0,10 & $\begin{array}{c}\mathbf{0} \\
(0,23)\end{array}$ & $\begin{array}{c}20 \\
(3,18)\end{array}$ & $\begin{array}{c}18 \\
(2,18)\end{array}$ & $\begin{array}{c}11 \\
(2,61)\end{array}$ & $\begin{array}{c}\mathbf{0} \\
(0,56)\end{array}$ & $\begin{array}{c}5 \\
(5,69)\end{array}$ & $\begin{array}{c}2 \\
(0,98)\end{array}$ & $\begin{array}{c}\mathbf{0} \\
(1,54)\end{array}$ \\
\hline & 0,20 & $\begin{array}{c}\mathbf{0} \\
(0,11)\end{array}$ & $\begin{array}{c}\mathbf{4 9} \\
(2,33)\end{array}$ & $\begin{array}{c}\mathbf{3 0} \\
(3,01)\end{array}$ & $\begin{array}{c}20 \\
(3.46)\end{array}$ & $\begin{array}{c}\mathbf{0} \\
(0,33)\end{array}$ & $\begin{array}{c}12 \\
(4,87)\end{array}$ & $\begin{array}{c}7 \\
(4,42)\end{array}$ & $\begin{array}{c}\mathbf{0} \\
(1,79)\end{array}$ \\
\hline
\end{tabular}

El valor entre paréntesis corresponde a la desviación estándar 
El perfil de la actividad antifúngica del extracto soluble en agua, muestra que es el más tolerable por los microorganismos de prueba y presenta una reducida actividad inhibitoria sobre $G$. trabeum y $T$. versicolor. Posiblemente esto se atribuye a la composición química de los extractos recuperados con este solvente. Investigaciones previas reportan que el agua fría y caliente, en la madera, tienen la propiedad de remover y extraer una gran cantidad de materiales orgánicos señalados como azúcares del duramen, representados mayormente por arabinosa, galactosa, glucosa, proteínas, trazas de xilosa y unidades de ácidos glucurónicos entre otros (Hillis, 1987; Sarampää y Höll, 1989; Sarampää y Nyberg 1989; Fischer y Höll, 1992; Viitanen et al., 1997; Willfor et al., 1999). Estos pueden ser fácilmente metabolizados por los microorganismos de deterioro de la madera e incrementar, más que inhibir, el crecimiento de los mismos, tal y como fue observado; ya que en muchas oportunidades, los microorganismos lograron crecer con mayor rapidez e incluso cubrir completamente la cápsula de Petri más rápido que los testigos. Con base en estas observaciones es posible predecir que los compuestos hidrofílicos presentes en el duramen de las especies evaluadas muestran un mínimo de efectos inhibitorios sobre los organismos que causan su deterioro.

Dentro de los extractos solubles en agua, el extracto de la especie Cartan fue, entre las cuatro especies, el más efectivo para el control de los microorganismos ( $49 \%$ de inhibición). Posiblemente debido a que dentro de su composición química, es el extracto que presenta la menor cantidad de carbohidratos no estructurales. En esa especie predomina mayor proporción del ingrediente activo, a diferencia de las especies Zapatero y Algarrobo las cuales presentaron un mínimo de efectos inhibitorios.
En la tabla 2 es evidente que los extractos acetónico y etanólico tienen la capacidad de extraer la mayor cantidad de compuestos biológicamente activos del duramen de las especies evaluadas, en comparación con el extracto recuperado en agua. Estas observaciones concuerdan con los resultados obtenidos en las investigaciones desarrolladas por French et al. (1983), Li et al. (1995) y Eloff (1998), quienes señalan que los compuestos biológicamente activos presentes en el duramen de las especies forestales, se encuentran distribuidos, tanto en el lumen, como en los microcapilares de la pared celular de las fibras. Estos en su mayoría son solubles en alcohol y acetona, siendo compuestos hidrofóbicos, de polaridad intermedia o compuestos poco polares. Adicionalmente, muestran pequeñas variaciones sobre la actividad biológica de los extractos acetónico y etanólico. Estas diferencias, evidencian el hecho de que el extracto en acetona presente una actividad biológica menor, posiblemente, debido a que este solvente tiene la capacidad de disolver y extraer muchos compuestos lipofílicos como grasas, ceras, gomas, resinas, ácidos grasos, materiales colorantes, fenoles, di- y triglicéridos, compuestos hidrofílicos altamente polares y compuestos fenólicos de bajo peso molecular (Rydholm, 1965; River et al., 1994; Gao y Breuil, 1995; Sutie y Osler, 1996; Eloff, 1998; Harju et al., 2003). Estos componentes pueden ser fácilmente asimilables por los microorganismos de prueba, de modo que la efectividad o acción de las sustancias biológicamente activas presentes en este extracto se vean levemente inhibidas por la presencia de tales sustancias (compuestos menos activos en mayores proporciones).

De acuerdo con investigaciones realizadas por Kurita y Kioke (1983) y Abdelrhafour et al. (1993), la actividad 
antimicrobial de diversos productos naturales se rige por el siguiente orden de mayores constituyentes: fenoles > alcoholes $>$ aldehídos $>$ ketonas $>$ éteres $>$ hidrocarburos. La mayor actividad microbiológica del extracto etanólico en comparación con los extractos acuoso y acetónico, podría estar en concordancia con estos resultados. De acuerdo con lo reportado por Sjöström (1981), Eaton y Hale (1993) y River et al. (1994), el etanol, como solvente, tiene la propiedad de extraer compuestos cíclicos, aromáticos, principalmente fenoles, polifenoles y compuestos relacionados, además de extraer fracciones de lignina de bajo peso molecular (Rydholm, 1965; Pereira et al., 1986); los cuales tienen una marcada influencia en la biorresistencia de la madera (Erikson et al., 1990; Eaton y Hale, 1993; Schultz y Darrel, 1997; Singh y Kim,1997). Adicionalmente, Abdelrhafour et al. (1993), Schultz et al. (1995) y Schultz y Darrel (1997), mencionan que la efectividad biológica de un extracto sobre otro, entre espe- cies, e inclusive dentro de una misma especie, puede deberse en gran parte a la presencia de componentes minoritarios específicos que actúan sinergéticamente con los compuestos más activos, incrementando la efectividad de cada extracto.

En la figura 1 se muestra el efecto fungicida existente entre los extractos etanólicos de las cuatro especies empleadas, a la menor concentración del extracto $(0,05 \% \mathrm{p} / \mathrm{v})$. En ella se observa el orden decreciente de actividad biológica-especies de los extractos asociado con T. versicolor y G. trabeum, donde las especies con mayores actividades fueron Puy y Cartan, y Algarrobo y Zapatero como las menos activas. Este mismo orden decreciente fue observado en el extracto en acetona. En cambio, en el extracto soluble en agua, las especies Cartan y Puy presentaron mayor actividad biológica frente a los dos microorganismos de prueba en comparación a las especies Algarrobo y Zapatero.

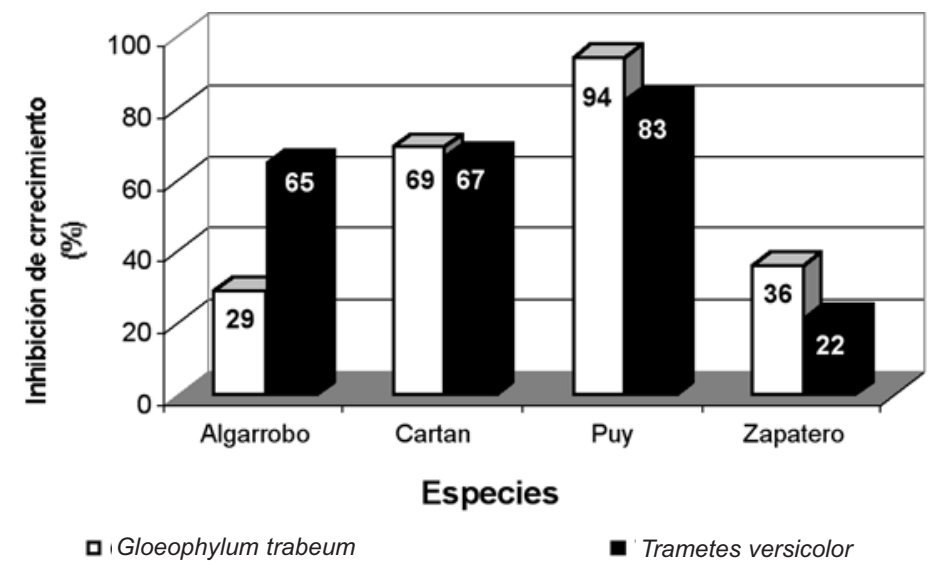

Figura 1. Actividad antifúngica del extracto etanólico $(0,05 \%)$, asociado a los dos microorganismos de prueba, al sexto día de incubación 
Dentro de las especies evaluadas se destaca por su amplia actividad biológica la especie Puy (Tabebuia serratifolia (Vahl.) Nicholson). Los resultados de estas evaluaciones concuerdan con investigaciones realizadas empleando diversas especies de la familia Bignoniaceae, pertenecientes a un grupo conocido como Lapachol, que incluye a la especie Puy, en las cuales se ha logrado demostrar que el extracto proveniente de estas especies se caracteriza por poseer propiedades insecticidas, antibacterianas, antifúngicas, anticancerígenas, antitumorales, antiinflamatorias, contra úlceras y gastritis, entre otras (Girard et al., 1987; Goel et al., 1987; Grazziotin et al., 1992), atribuidos a la presencia en este género (Tabebuia), de compuestos conocidos comúnmente como lapachol y sus derivados (quinonas de amplio espectro). La presencia de sustancias biológicamente activas y de elevada importancia en la especie Puy, hace de ésta una fuente promisoria de posibles fungicidas naturales, por lo que amerita profundizar en el aislamiento e identificación de las sustancias responsables de dicha bioactividad.

\section{CONCLUSIONES}

Los extractos crudos provenientes del duramen de las especies Tabebuia serratifolia, Peltogyne porphyrocardia, Hymenaea courbaril y Centrolubium paraense, evaluados en la investigación, mostraron actividad antifúngica frente a los hongos utilizados en el ensayo. El extracto acuoso evidenció ser el más tolerante por los microorganismos de prueba, posiblemente debido a su composición química. Los extractos acetónicos y etanólicos al 0,10\% y 0,20\%, presentaron un comportamiento similar en el control de G. trabeum y $T$. versicolor. Sin embargo, el extracto etanólico del duramen de $T$. serratifolia, mostró supe- rioridad al controlar el desarrollo total del micelio, de los microorganismos a una concentración de $0,20 \%$.

\section{RECONOCIMIENTOS}

Los autores expresan su agradecimiento al Grupo de Investigación en Conservación de la Madera, del Laboratorio Nacional de Productos Forestales de la ULA, por el suministro de los microorganismos de prueba y al FONACIT por el financiamiento otorgado a través del proyecto Pem-2001001639.

\section{REFERENCIAS}

Abdelrhafour, T.N., Lattaoui y A. Errifi 1993. Composition and antimicrobial activity of the essential oils of Thymus broussonettii, T. zygis and $T$. satureioides. Journal of Essential Oil Research 5:45-53.

Ajaiyeoba, E.A., Rahman y I. Choudhary 1998. Preliminary antifungal and cytotoxicity studies of extracts of Ritchiea capparoides var. longipedicellata. Journal of Ethnopharmacology 62(3):243-246.

Bhargava A., A. Srivastava y V. Kumbhare. 1998. Antifungal activity of polyphenolic complex of Acacia nilotica bark. The Indian Forester 124 (5):292-298.

Curling, S., J. Winandy y C. Clausen. 2000. An experimental method to simulate incipient decay of wood by basidiomycete fungi. The International Research Group on Wood Preservation. Document núm. IRG/WP 00-20200.

Deon, G. 1983. About the relations between the natural durability of some tropical species and their 
extractives content. The International Research Group on Wood Preservation. Document núm. IRG/ WP/ 12018.

Eaton, R. y M. Hale. 1993. Wood: Decay, Pests and Protection. Chapman and Hall, Londres. 546 p.

Eloff, J. 1998. Which extractant should be used for the screening and isolation of antimicrobial components from plants? Journal of Ethnopharmacology 60(1):18

Erikson, K., R. Blanchette y P. Ander 1990. Microbial and enzymatic degradation of wood and wood components. Springer Verlag, Berlin. $407 \mathrm{p}$.

Fischer, C. y W. Höll. 1992. Food reserves of scots pine (Pinus silvestris L.). II. Seasonal changes and radial distribution of carbohydrate and fat reserves in pine wood. Trees structures and Function 6(3):147-155.

French, J., P. Robinson y J. Creffield. 1983. Bioassays of extracts from scaly ash (Ganophyllum falcatum B1) against the subterranean termite Coptotermes acinaciformis (Froggatt). The International Research Group on Wood Preservation. Document IRG/WP/1206.

Gao, Y. y C. Breuil. 1995. Wood extractives as carbon sources for staining fungi in the sapwood of Lodgepole pine and Trembling aspen. The International Research Group on Wood Preservation. Document IRG/WP 95-10098.

García, C., E. Correa y N. Rojas. 1995. Estudio fitoquímico preliminar $\mathrm{y}$ evaluación de la actividad antimicrobiana de algunas plantas superiores colombianas. Revista Colombiana de Ciencias Químico-Farmaceúticas 23: 42-48.

Gierlinger, N., D. Jacques, M. Grabner, R. Wimmer, M. Schwanninger, P. Rozenberg y L. Pâques. 2004. Colour of larch heartwood and relationships to extractives and brownrot decay resistance. Trees 18(1):102-108

Girard, M., J. Ethier, D. Kindack, B. Dawson y D. Awang. 1987. Mass spectral 1. Characterization of naphthoquinones related to Lapachol. Journal of Natural Products 50(6):1149-115

Goodell, B., J. Jellison, J. Liu, G. Daniel, A. Paszczynski, F. Fekete, S. Krishnamurthy, L. Jun y G. Xu.1997. Low molecular weight chelators and phenolic compounds isolated from wood decay fungi and their role in the fungal biodegradation of wood. Journal of Biotechnology 53(2):133162.

Goel, R., N. Pathak, M. Biswas, V. Pandey y A. Sanyal. 1987. Effect of Lapachol, a naphthaquinone isolated from Tectona grandis, on experimental peptic ulcer and gastric secretion. Journal Pharmacia and Pharmacology 39(2):138-140.

Gopalakrishnan, G., B. Banumathi y G. Suresh. 1997. Evaluation of the antifungal activity of natural xanthones from Garcinia mangostana and their synthetic derivates. Journal of Natural Products 60 (5): 519-524.

Grazziotin, J., E. Schapoval, C. Chaves, J. Gleye y A. Henriques. 1992. Phytochemical and analgesic investigation of Tabebuia chrsotricha. Journal of Ethnopharmacology $36(2): 249-251$ 
Harju, A., M. Venäläinen, S. Anttonen, H. Viitanen, P. Kainulainen, P. Saranpää y E. Vapaavuori. 2003. Chemical factors affecting the brown-rot decay resistance of Scots pine heartwood. Trees 17(2):263-268.

Hillis, W. 1987. Heartwood and tree exudates. Syracuse, New York. EUA. Springer Series in Wood Science. 267 p.

IFLA. 1987. Maderas Comerciales de Venezuela: Algarrobo (Hymeneae courbaril). Instituto Forestal Latinoamericano, ficha técnica núm. 6 . Mérida, Venezuela. 29 p.

Jansser, A., J. Senefief y A. Baerherm. 1987. Antimicrobial activity of essential oils: A 1976-1986 literature review. Aspects of the test methods. Planta Medica 53:395-398.

Kennedy, M., H. Jiang y L. Stephens. 2000. Natural durability transfer from sawmill residues of white cypress (Callistris glaucophylla); 1: Optimisation of the extraction conditions. The International Research Group on Wood Preservation. Document núm. IRG/ WP/ 00-30238.

Kurita, N. y S. Koike. 1983. Synergetic antimicrobial effect of etanol, sodium chloride, acetic acid and essential oil components. Agric. Biolo. Chem 47(1):67-75.

Li, E., A. Clark y C. Hufford. 1995. Antifungal evaluation of Psudolaric acid $\mathrm{B}$, a major constituent of Pseudolarix kaempeferi. Journal of Natural Products 58(1):57-67.

Mora, N. y O. Encinas. 2001. Evaluación de la durabilidad natural e inducida de Pterocarpus acapulcensis, Tabebuia serratifolia y Pinus caribaea, en condiciones de laboratorio.
Revista Forestal Venezolana 45(1):23-31.

Mora, N., O. Encinas y L. Villarreal. 2004. Biodegradación de maderas venezolanas o de la Red Nacional de Cementerios de Estacas. Memorias IV Congreso Forestal Venezolano: Bosque Vida y Desarrollo. $60 \mathrm{p}$.

Papinutti, V., L. Diorio y F. Forchiassin. 2003. Degradación de la madera de álamo por Fomes sclerodermus: Producción de enzimas ligninolíticas en aserrín de álamo y cedro. Revista Iberoamericana de Micología 20:1620.

Pereira, H., M. Oliveira y I. Miranda. 1986. Kinetics of etanol-water pulping and pulp properties of Eucalyptus globulus Lab. Appita 39 (6):15-20.

River, B., C. Vick y R. Gillespie. 1994. Wood as an adherend. In: D. Minford, ed. Treatise on adhesion and adhesives. New York. Chapter 1. $230 \mathrm{p}$.

Rydholm, S. 1965. Pulping Processes. John Wiley \& Sons, Inc. Nueva York. $1269 \mathrm{p}$.

Saranpää, P. y W. Höll. 1989. Soluble carbohydrates of Pinus sylvestris $\mathrm{L}$. sapwood and heartwood. Trees structures and Function 3(3): 138143.

Saranpää, P. y H. Nyberg. 1989. Lipids and sterols of Pinus sylvestris L. sapwood and heartwood. Trees structures and Function 1(2): 82-87.

Schultz, T., W. Harms, T. Fisher, K. McMurtrey y O. Nicholas. 1995. Durability of Angiosperm heartwood: the importance of extractives. Holzforschung 49:29-34. 
Schultz, T. y D. Darrel. 1997. Susceptibility of angiosperm sapwood to whiterot fungal colonization and subsequent degradation: A hypothesis. The International Research Group on Wood Preservation. Document núm. IRG/ WP/ 97-10211.

Singh, A. y Y. Kim. 1997. Biodegradation of wood in wet environments: a review. The International Research Group on Wood Preservation. Document núm. IRG/ WP/ 97-10217.

Sjöström, E. 1981. Wood Chemistry Fundamental and Applications. Orlando, Florida.

Suttie, E. y R. Orsler. 1996. The influence of the natural extractives of Opepe (Nauclea diderrichii) and African padauk (Pterocarpus soyauxii) timbers on their durability. The International Research Group on Wood Preservation. Document núm. IRG/ WP/ 96-30098.

TAPPI. 1998. Preparation of wood for chemical analysis. Technical Association of Standard Pulp and Paper Industry. NORMT 264 om-97.

TAPPI. 1998. Water solubility of wood and pulp. Technical Association of Standard Pulp and Paper Industry. NORMT 207 om-93.

Troya, M., A. Navarrete, F. Rubio, M. Yuste, C. Rodríguez, D. Muñoz y F. Linares. 1999. Proposal for a simple methodology for the evaluation of the preventive efectiveness of protectors applied in superficial treatmens against basidiomycetes fungi. The International Research Group on Wood Preservation. Document núm. IRG/ WP/ 99-20167.

Uzcátegui, M., O. Encinas, A. Briceño y N. Mora. 2004. Durabilidad natural de
Swietenia macrophylla de plantaciones. Memorias IV Congreso Forestal Venezolano: Bosque Vida y Desarrollo. 65 p.

Velásquez, J. 2000. Durabilidad inducida de la madera de Pino caribe (Pinus caribaea var. hondurensis) con extractos de algunas latifoliadas de durabilidad natural elevada. Tesis, Magister Scientiae. ULA, Mérida. Venezuela. $51 \mathrm{p}$.

Velásquez, J., O. Encinas, L. Rojas y A. Usubillaga. 2004. Durabilidad natural del duramen de Tabebuia serratifolia. Memorias IV Congreso Forestal Venezolano: Bosque Vida y Desarrollo. 67 p.

Viitanen, H., L. Paajanen, P. Saranpää y P. Viitaniemi. 1997. Durability of larch (Larix sp.) wood against brownrot fungi. The International Research Group on Wood Preservation. Document núm. IRG/ WP/ 97-10228.

Winandy, J. y J. Morell. 1993. Relationship between incipient decay, strength, and chemical composition of douglas-fir heartwood. Wood and Fiber Science 25(3): 278-288.

Willför, S., R. Sjöholm y B. Holmbom. 1999. Isolation and characterization of water-soluble arabinogalactans from the heartwood of Norway spruce and Scots pine. In $10^{\mathrm{TH}}$ International Symposium on Wood and Pulping Chemistry. Yokohama, Japan. Tomo II, pp 32-34.

Wong, A. y A. Singh. 1997. Microbial decay in an extremely durable Malaysian hardwood belian (Eusideroxylon zwageri) - an overview. The International Research Group on Wood Preservation. Document núm. IRG/ WP/ 97-10216. 
Manuscrito recibido el 1 de abril de 2005.

Aceptado el 12 de diciembre de 2005.

Este documento se debe citar como:

Velásquez, J., M.E. Toro, L. Rojas y O. Encinas. 2006. Actividad antifúngica in vitro de los extractivos naturales de especies latifoliadas de la Guayana Venezolana. Madera y Bosques 12(1):51-61. 